Original Article (short paper)

\title{
The intensities of various forms of physical activity in physical education programs offered by universities for female students
}

\author{
Robert Podstawski ${ }^{1}$ (D), Marta A. Żurawik² (D) , Cain C. T. Clark ${ }^{3}$ (D) , Ferenc Ihasz $^{4}$ (D), Piotr Żurek ${ }^{5}$ (1) \\ ${ }^{1}$ University of Warmia and Mazury in Olsztyn, Faculty of Geoengineering, Department of Tourism, Recreation and \\ Ecology, Olsztyn, Poland; ${ }^{2}$ Independent Researcher, Wigan, UK; ${ }^{3}$ Coventry University, Faculty of Health and Life \\ Sciences, Coventry, UK; ${ }^{4}$ Eötvös Lóránd University, Faculty of Psychology and Pedagogy, \\ Institute of Sports Sciences, Szombathely, Hungary; ${ }^{5}$ Poznan University of Physical Education, \\ Department of Physical Education in Gorzow Wielkopolski, Poland \\ Associate Editor: Sandro Carnicelli, University of the West of Scotland, Scotland/UK
}

\begin{abstract}
Aim: This study aimed to evaluate the effectiveness of various forms of physical activity (PA) among female students in physical education (PE) programs offered by universities in Poland, Hungary, and the United Kingdom. Methods: Two hundred full-time female university students (mean age: 19.93 \pm 0.82 ) enrolled in various PA programs. The participants' anthropometric traits were measured, and their body composition parameters were determined with the InBody analyzer. Based on the students' physiological parameters, the effectiveness of various types of PA was measured with Suunto Ambit3 peak heart rate monitors during 60 minutes of physical exertion. Results: The average values of body mass index (BMI), body fat mass (BFM), percent body fat (PBF), waist-hip ratio (WHR), visceral fat level $(\mathrm{VFL})$, and obesity degree were significantly $(\mathrm{p}<0.05)$ lower among students who participated in jogging followed by sauna (JFBS), performed martial arts and attended general physical education (PE) classes. Physiological parameters were the highest in the martial art group, followed by JFBS and swimming groups, and they were significantly $(\mathrm{p}<0.05)$ higher than the values recorded in other PA groups (golf, aerobics, general PE classes, cycling, and individual training). Physiological parameters were significantly $(\mathrm{p}<0.05)$ lower among students who played golf and trained individually. Conclusions: Martial arts, JFBS, and swimming were the most effective types of PA among female university students. Students performing martial arts and JFBS had relatively lower body fat levels, whereas students who practiced swimming had the highest body fat levels in the population sample.
\end{abstract}

Keywords: female university students; effectiveness; physical activity forms; body composition; physiological parameters

\section{Introduction}

Physical activity (PA) decreases the risk of overweight and obesity, lowers total cholesterol levels, and blood pressure ${ }^{1}$. According to the American College of Sports Medicine², adults should perform at least 30 minutes of moderate-intensity cardiorespiratory exercise training five times per week (total of $\geq 150 \mathrm{~min} \cdot \mathrm{wk}^{-1}$ ), or at least 20 minutes of vigorous-intensity cardiorespiratory exercise training three times per week (total of $\geq 75 \mathrm{~min} \cdot \mathrm{wk}^{-1}$ ), or a combination of moderate- and vigorous-intensity exercise to achieve a total energy expenditure of $\geq 500-1000 \mathrm{MET} \cdot \mathrm{min} \cdot \mathrm{wk}^{-1}$. In addition, resistance exercises targeting the major muscle groups, neuromotor exercises for improved balance, agility, and coordination, and flexibility exercises focusing on the major muscle-tendon groups are also recommended and should be performed at least twice per week.

The growing scientific awareness of active lifestyle contributions to overall health has prompted educational reforms targeting PE curricula worldwide and has ushered changes in the existing approach to PE and health education.
In recent decades, PA programs in higher education have changed and no longer resemble obligatory physical education (PE) classes from the past. Many PA programs have an informal character with the aim of encouraging students to undertake any type of exercise in their free time. Also, new forms of PA are being introduced to the universities in order to increase the attractiveness of PE programs, including fitness-based activities, such as aerobics and jazz gymnastics, as well as activities that are generally popular among young adults, such as snowboarding, and high intensity-functional training (HIFT), including high-intensity interval training (HIIT), high-intensity circuit training (HICT) and Crossfit ${ }^{3,4}$.

However, the general decrease in the number of obligatory PE classes in higher education contributes to a further decline in the PA levels of university students ${ }^{5,6}$. The low levels of PA among young adults contribute to the frequency of overweight and obesity, which has been reported to be the highest in the population ages 18 to $29^{7}$. University The Youth Risk Behavior Survey ${ }^{8}$ demonstrated that $55 \%$ of high school seniors have adequate PA levels, whereas less than $40 \%$ of university students 
meet the recommended standard ${ }^{9}$. A similar decrease in PA levels between adolescence and adulthood has been reported in the literature in particular among the first year students ${ }^{10,11}$. Interestingly, university students were reported to more likely to gain weight than their peers who were not in higher education ${ }^{12}$. A body of research suggests that the percentage of sedentary university students varies across countries, from $23 \%$ in Western Europe and the USA to 30\% in Central and Eastern Europe, 39\% in the Mediterranean region, $42 \%$ in the Asia-Pacific region, and $44 \%$ in developing countries ${ }^{13}$. University students generally gain weight due to low levels of PA as well as an excessive caloric intake. First-year students gain approximately $4 \mathrm{lbs}$ in the course of 3-12 months ${ }^{14}$ and tend to maintain the gained weight ${ }^{15}$ or even continue to gain weight in successive years of higher education ${ }^{16}$. Additionally, in most cases, excess weight contributes to an increase in fat mass ${ }^{15,17,18}$. Therefore, regular participation in PA and PE programs is vital for young adults in higher education. However, standard PE classes may not be a realistic requirement for full-time university students to encourage regular participation in sports and PA due to busy academic schedules and limited time to complete courses. Furthermore, the effectiveness of standard PE classes has also been questioned in the literature ${ }^{19,20}$. For instance, Podstawski et al. ${ }^{21}$ proposed that independent training could be an attractive alternative for students who were keen on maximizing the outcomes of exercise programs in a limited period. Nevertheless, the changes in approach to PA in higher education raise the question of the meaning and relevance of PE for young people, as well as the quality of the provided programs ${ }^{22}$. Therefore, the purpose of this study is to investigate the effectiveness of PA programs in higher education. The study focuses on evaluating the body composition parameters of the female university students in three countries (Poland, Hungary, and the United Kingdom) in order to determine the relationship between individual fitness traits and choice of PA programs.

\section{Methods}

\section{Participants}

This study was conducted according to the recognised ethical standards of the Helsinki Declaration.

Prior to the commencement of this study, all procedures involving human participants were granted ethical approval by the research ethics committee of the University of Warmia and Mazury in Olsztyn, Poland (39/2011). This cross-sectional study was conducted with a total of 200 full-time female students ( $M=19.93 \pm 0.82$ years old) from six universities, two in Poland, two in Hungary, and two in the United Kingdom. Two hundred participants were divided into eight groups. Each group consisted of twenty-five students, who enrolled in various PA programs, such as golf, martial arts, jogging followed by sauna (JFBS), swimming, traditional Physical Education (PE) classes, cycling, and individual training (any type of PA without formal supervision). Study participants enrolled online in selected PA programs at the beginning of every semester in order to obtain credits. In Hungarian and British universities, students were also given credits for individual training, which took place at a specified location and time and was registered by PE teachers. All participants were randomly selected in order to produce coherent groups that represented different types of PA. To guarantee equal sample size, up to five students could participate in a PA program taught by one instructor, and every form of PA had to be performed by students from at least two countries. All students gave their informed consent to participate in the study and were assured of confidentiality and anonymity and the right to withdraw from the study at any time. Those who did not wish to participate in the study were replaced by other randomly selected candidates. All students were in good health, had no history of blood diseases or diseases affecting biochemical and biomechanical factors, and did not take any medication or nutritional supplements.

\section{Procedures}

Data collection was conducted in April 2019. The following procedures were followed: 1) body height was measured to the nearest $1.0 \mathrm{~mm}$ with a calibrated WB-150 medical scale with a stadiometer (ZPU Tryb Wag, Poland) and the Martin anthropometer according to standardized guidelines; 2) body mass was measured to the nearest $0.1 \mathrm{~kg}$; 3) BMI and body composition parameters (weight, total body water-TBW, protein, minerals, body fat mass - BFM, fat-free mass - FFM, skeletal muscle mass - SMM, body mass index - BMI, percent body fat - PBF, waist-hip ratio - WHR, visceral fat level - VFL, and levels of obesity) were determined by bioelectrical impedance ${ }^{23}$ with the InBody 720 body composition analyser. All anthropometric measurements and body mass composition parameters were analyzed immediately before the physical training, in the state of rest.

During all PA programmes, the participants wore pulsometers on their wrists, and Suunto Ambit3 Peak HR monitor sensors were attached to their chests, which are commonly used in the studies evaluating the intensity of various forms of $\mathrm{PA}^{24,25}$, and are considered accurate and reliable ${ }^{26,27}$. Every pulsometer was programmed to the female sex, year of birth, body mass, and PA levels. Participants activated the pulsometers 60 minutes before each PA training and deactivated after completing the PA sessions.

HR monitors recorded estimated values of physiological parameters, including heart rate $\left(\mathrm{HR}_{\text {min, avg, max }}\right)$, recovery time, peak training effect (PTE), energy expenditure, oxygen uptake $\left(\mathrm{VO}_{2 \text { avg, max }}\right)$, excess post-exercise oxygen consumption (EPOC ${ }_{\text {avg, }}$ peak $)$, respiratory rate $\left(\mathrm{RR}_{\mathrm{avg}, \max }\right)$ and training parameters, such as recovery, peak training effect - PTE, exercise intensity: low ( $<107 \mathrm{bpm})$, moderate (107-124 bpm), high (125-141 bpm), very high (142-159 bpm), maximal ( $\geq 160 \mathrm{bpm})^{24,25}$. Physiological parameters were measured during 60-minute training sessions. HR monitors were switched on when PA training started and immediately switched off afterward by saving the results in Movescount Suunto application.

All participants visited a dry sauna during weekly PE classes 
on the same day, in the same location, and over the same period, to minimize the effect of diurnal variation on the results ${ }^{28}$. Every participant attended one 15-minute sauna session (temperature: $90^{\circ} \mathrm{C}$; relative humidity: $14-16 \%$ ) remaining in a sitting position. The results from sauna sessions offered additional data for analyses and comparisons with the results from other forms of PA, as they assisted in determining the intensity of physiological processes in jogging vs sauna.

\section{Statistical analysis}

The participants were divided into coherent groups of 25 subjects each. Anthropometric features, body composition, and physiological parameters were analyzed using the Statistica PL v. 13.5. The basic descriptive statistics, such as mean values, standard deviation, and the minimum and maximum values of the measured parameters were conducted for each group. It must be noted that the data in column 7 reports the results for the sauna session, as a post jogging activity, and was not included in the comparative analyses. One-way ANOVA with LSD 'post hoc' was used to assess the mean differences between the groups. The statistical significance was set at a level of $\mathrm{p}<0.05$. We computed achieved power, a posteriori, and based on the sample size, an observed effect size of 0.39 (for BMI), and an alpha error probability of 0.05 , a power $(1-$ beta probability) of 0.91 was attained.

\section{Results}

The study results are presented in a table format, where the measured parameters for each PA group are presented separately in columns. The differences between the PA groups are presented in the last column, from maximum to minimum values. The non-significant results are separated by a comma, and the significant results are separated by ' $>$ '.

The study results showed no significant differences in age, height, body score, and target weight among the participants in different PA groups. With regards to the average body mass, the scores were significantly higher $(\mathrm{p}<0.05)$ among golf players and significantly lower $(p<0.05)$ in the jogging group. Furthermore, female golf players had significantly higher values of proteins, minerals, BFM, FFM, and SMM $(p<0.05)$ than other participants. Concerning the values of BFM, FFM, SMM, BMI, PBF, VFL, a significant difference was observed among joggers $(p<0.05)$ who also had the lowest obesity level compared to other groups. The examination of the BMI showed that all participants were a healthy weight (BMI between 18.5 and 24.9), with the swimming group scoring the highest BMI of $24.7 \mathrm{~kg} / \mathrm{m}^{2}$. The analysis of WHR suggested android obesity and high health risk (WHR $\geq .87$ ) among participants in aerobics, golfing, and swimming groups. The moderate health risk (WHR $\leq .85)$ was recorded in cycling and individual training groups.

In Table 2, the analyses of the physiological parameters revealed the $\mathrm{HR}_{\text {avg }}$ value of $82.81( \pm 7.98) \mathrm{bpm}$ was significantly higher among the martial arts group $(\mathrm{p}<0.05)$. Furthermore, significantly higher $(\mathrm{p}<0.01)$ values of $\mathrm{HR}_{\mathrm{avg}}$ were also observed in JFBS $(80.96 \pm 10.52 \mathrm{bpm})$ and swimming $(81.64 \pm 6.66)$ groups in comparison to the others. In contrast, the significantly $(\mathrm{p}<0.05)$ lower values of $\mathrm{HR}_{\text {avg }}$ were recorded in golfing groups $(67.24 \pm 4.94 \mathrm{bpm})$ and participants who trained individually (70.64 $\pm 6.82 \mathrm{bpm})$. Regarding values of $\mathrm{HR}_{\max }$, the significantly $(\mathrm{p}<0.05)$ higher results were among students practicing martial arts (159.19 $\pm 10.88 \mathrm{bpm})$ and JFBS $(156.48 \pm 10.57 \mathrm{bpm})$, whereas the significantly lower values $(\mathrm{p}<0.05)$ were recorded in a golfing group (114.32 $\pm 10.06 \mathrm{bpm})$. Correspondingly, students who participated in martial arts and JFBS observed having the highest energy expenditure $(638.38 \pm 43.91 \mathrm{kcal}$ and $595.88 \pm 51.62$ $\mathrm{kcal}$ respectively), the highest values of $\mathrm{VO}_{2 \text { avg }}(22.81 \pm 1.74 \mathrm{~mL} /$ $\mathrm{kg} / \mathrm{min}$ for martial arts, $20.64 \pm 2.16 \mathrm{~mL} / \mathrm{kg} / \mathrm{min}$ for JFBS), $\mathrm{VO}_{2}$ max $(33.31 \pm 3.97 \mathrm{~mL} / \mathrm{kg} / \mathrm{min}$ for martial arts, $32.56 \pm 4.82 \mathrm{~mL} / \mathrm{kg} /$ min for JFBS), EPOC $(25.88 \pm 4.66 \mathrm{~mL} / \mathrm{kg}$ for martial arts, $21.13 \pm 6.8 \mathrm{~mL} / \mathrm{kg}$ for JFBS $),$ EPOC $_{\text {max }}(47.12 \pm 13.12 \mathrm{~mL} / \mathrm{kg}$ for martial arts, $40 \pm 9.07 \mathrm{~mL} / \mathrm{kg}$ for JFBS $), \mathrm{RR}_{\mathrm{avg}}(23.42 \pm 1.21 \mathrm{brpm}$ for martial arts, $22.76 \pm 1.42$ brpm for JFBS), RR max $_{\text {ax }}(35.23 \pm 4.16$ brpm for martial arts, $35.12 \pm 4$ brpm for JFBS). The analyses of exercise intensity in minutes showed that intensity of $107 \mathrm{bpm}$ which was considered easy was significantly longer $(p<0.05)$ in the golfing group (54:18 $\pm 6: 27 \mathrm{~min})$. The moderate exercise intensity between 107 and $124 \mathrm{bpm}$ persisted the longest $(\mathrm{p}<0.05)$ in the swimming group (26:54 $\pm 9: 55 \mathrm{~min})$. The exercise intensity classified as difficult (125-141 bpm), very difficult (142-159 bpm), and maximal effort (over $160 \mathrm{bpm}$ ) continued for significantly longer periods $(\mathrm{p}<0.05)$ among martial arts students and joggers in comparison to other PA groups.

Table 1 - Somatic traits and body composition parameters in women performing various types of physical activity

\begin{tabular}{|c|c|c|c|c|c|c|c|c|c|c|}
\hline \multirow[t]{2}{*}{ Traits } & Golf & Martial arts & Aerobic & Jogging & Swimming & General P.E. & Sauna & Cycling & $\begin{array}{c}\text { Individual } \\
\text { training }\end{array}$ & Differences \\
\hline & 1 & 2 & 3 & 4 & 5 & 6 & 7 & 8 & 9 & \\
\hline Age [years] & $\begin{array}{c}20.29 \pm 1.46 \\
(18.3-24)\end{array}$ & $\begin{array}{c}20.35 \pm 1.85 \\
(18-24)\end{array}$ & $\begin{array}{c}19.99 \pm 2.54 \\
(18-31)\end{array}$ & $\begin{array}{c}19.77 \pm 1.53 \\
(18-23)\end{array}$ & $\begin{array}{c}20.29 \pm 1.62 \\
(18.1-24)\end{array}$ & $\begin{array}{c}20.28 \pm 1.77 \\
(18.1-24)\end{array}$ & $\begin{array}{c}20.1 \pm 1.83 \\
(18-24)\end{array}$ & $\begin{array}{c}20 \pm 1.29 \\
(18.1-23)\end{array}$ & $\begin{array}{c}20.36 \pm 0.86 \\
(19-22)\end{array}$ & $9,2,5,1,6,7,8,3,4$ \\
\hline Body height [cm] & $\begin{array}{c}167.28 \pm 4.93 \\
(158-176)\end{array}$ & $\begin{array}{c}166.31 \pm 6.08 \\
(156-179)\end{array}$ & $\begin{array}{c}166.64 \pm 5.65 \\
(160-179)\end{array}$ & $\begin{array}{c}165.04 \pm 5.82 \\
(152-174)\end{array}$ & $\begin{array}{c}164.4 \pm 5.49 \\
(155-175)\end{array}$ & $\begin{array}{c}167.04 \pm 5.13 \\
(158-179)\end{array}$ & $\begin{array}{c}165.72 \pm 5.54 \\
(156-178)\end{array}$ & $\begin{array}{c}167.36 \pm 5.66 \\
(159-183)\end{array}$ & $\begin{array}{c}165.48 \pm 3.87 \\
(159-172)\end{array}$ & $8,1,6,3,2,7,9,4,5$ \\
\hline Body mass $[\mathrm{kg}]$ & $\begin{array}{c}69.2 \pm 9.52 \\
(54.4-86)\end{array}$ & $\begin{array}{l}58.34 \pm 6.73 \\
(46.6-73.6)\end{array}$ & $\begin{array}{l}65.83 \pm 16.16 \\
(47.3-115.9)\end{array}$ & $\begin{array}{l}52.88 \pm 6.83 \\
(38.1-67.3)\end{array}$ & $\begin{array}{l}66.72 \pm 12.9 \\
(48.4-96.5)\end{array}$ & $\begin{array}{l}59.35 \pm 8.74 \\
(46.6-84.8)\end{array}$ & $\begin{array}{c}66.26 \pm 11.73 \\
(43.4-85.9)\end{array}$ & $\begin{array}{l}63.26 \pm 10.48 \\
(50.2-104.9)\end{array}$ & $\begin{array}{c}60.17 \pm 11.17 \\
(42.2-86.3)\end{array}$ & $1>5,7,3,8,9,6,2>4$ \\
\hline $\begin{array}{l}\text { TBW (Total Body } \\
\text { Water) [L] }\end{array}$ & $\begin{array}{l}34.72 \pm 3.29 \\
(28.6-39.7)\end{array}$ & $\begin{array}{l}31.73 \pm 3.76 \\
(24.7-39.9)\end{array}$ & $\begin{array}{l}32.97 \pm 4.52 \\
(26.3-44.2)\end{array}$ & $\begin{array}{l}29.88 \pm 3.52 \\
(23.1-37.8)\end{array}$ & $\begin{array}{l}32.44 \pm 3.36 \\
(26.6-37.6)\end{array}$ & $\begin{array}{l}32.27 \pm 3.37 \\
(26.1-39.9)\end{array}$ & $\begin{array}{l}33.25 \pm 4.3 \\
(24.7-39.6)\end{array}$ & $\begin{array}{c}32.88 \pm 3.95 \\
(27-47.3)\end{array}$ & $\begin{array}{c}30.69 \pm 2.73 \\
(26-37)\end{array}$ & $1,7>3,8,5,6,2,9>4$ \\
\hline
\end{tabular}




\begin{tabular}{|c|c|c|c|c|c|c|c|c|c|c|}
\hline Proteins $[\mathrm{kg}]$ & $\begin{array}{l}9.32 \pm 0.89 \\
(7.7-10.7)\end{array}$ & $\begin{array}{l}8.52 \pm 0.99 \\
(6.7-10.7)\end{array}$ & $\begin{array}{l}8.83 \pm 1.22 \\
(7.1-11.8)\end{array}$ & $\begin{array}{c}8 \pm 0.99 \\
(6.1-10.2)\end{array}$ & $\begin{array}{l}8.73 \pm 0.92 \\
(7.3-10.2)\end{array}$ & $\begin{array}{l}8.66 \pm 0.91 \\
(7.1-10.7)\end{array}$ & $\begin{array}{l}8.95 \pm 1.18 \\
(6.7-10.7)\end{array}$ & $\begin{array}{l}8.81 \pm 1.04 \\
(7.3-12.6)\end{array}$ & $\begin{array}{c}8.24 \pm 0.76 \\
(7-10)\end{array}$ & $1>7,3,8,5,6,2,9,4$ \\
\hline Minerals $[\mathrm{kg}]$ & $\begin{array}{c}3.47 \pm 0.37 \\
(2.87-4.05)\end{array}$ & $\begin{array}{c}3.1 \pm 0.41 \\
(2.41-4.05)\end{array}$ & $\begin{array}{l}3.26 \pm 0.48 \\
(2.64-4.62)\end{array}$ & $\begin{array}{c}2.88 \pm 0.36 \\
(2.08-3.62)\end{array}$ & $\begin{array}{c}3.2 \pm 0.4 \\
(2.64-3.81)\end{array}$ & $\begin{array}{l}3.18 \pm 0.37 \\
(2.43-3.94)\end{array}$ & $\begin{array}{l}3.28 \pm 0.49 \\
(2.41-4.05)\end{array}$ & $\begin{array}{c}3.26 \pm 0.43 \\
(2.64-4.88)\end{array}$ & $\begin{array}{l}3.02 \pm 0.27 \\
(2.53-3.81)\end{array}$ & $1>7,3,8,5,6,2,9,4$ \\
\hline $\begin{array}{l}\text { BFM (Body Fat } \\
\text { Mass) }[\mathrm{kg}]\end{array}$ & $\begin{array}{c}21.67 \pm 6.86 \\
(5.3-34.8)\end{array}$ & $\begin{array}{l}14.98 \pm 3.94 \\
(10.2-26.5)\end{array}$ & $\begin{array}{c}20.76 \pm 11.46 \\
(8.5-55.3)\end{array}$ & $\begin{array}{c}12.11 \pm 4.33 \\
(6-22.3)\end{array}$ & $\begin{array}{c}22.36 \pm 10.18 \\
(9.6-50.2)\end{array}$ & $\begin{array}{c}15.25 \pm 6.49 \\
(5.3-34.4)\end{array}$ & $\begin{array}{c}20.77 \pm 8.08 \\
(6.2-41.2)\end{array}$ & $\begin{array}{l}18.31 \pm 6.03 \\
(10.4-40.1)\end{array}$ & $\begin{array}{c}18.22 \pm 8.83 \\
(5.7-41.2)\end{array}$ & $5,1,7,3>8,9>6,2>4$ \\
\hline $\begin{array}{l}\text { FFM (Fat Free } \\
\text { Mass) }[\mathrm{kg}]\end{array}$ & $\begin{array}{l}47.53 \pm 4.54 \\
(39.2-54.5)\end{array}$ & $\begin{array}{l}43.35 \pm 5.17 \\
(33.8-54.5)\end{array}$ & $\begin{array}{l}45.07 \pm 6.21 \\
(36.1-60.6)\end{array}$ & $\begin{array}{l}40.77 \pm 4.84 \\
(31.3-51.6)\end{array}$ & $\begin{array}{l}44.37 \pm 4.67 \\
(36.5-51.6)\end{array}$ & $\begin{array}{l}44.1 \pm 4.64 \\
(35.6-54.5)\end{array}$ & $\begin{array}{l}45.49 \pm 5.97 \\
(33.8-54.4)\end{array}$ & $\begin{array}{l}44.95 \pm 5.41 \\
(36.9-64.8)\end{array}$ & $\begin{array}{l}41.94 \pm 3.74 \\
(35.5-50.8)\end{array}$ & $1>7,3,8,5,6,2>9,4$ \\
\hline $\begin{array}{l}\text { SMM (Skeletal } \\
\text { Muscle Mass) [kg] }\end{array}$ & $\begin{array}{l}26.15 \pm 2.69 \\
(21.2-30.4)\end{array}$ & $\begin{array}{c}23.7 \pm 3.05 \\
(18.1-30.4)\end{array}$ & $\begin{array}{l}24.69 \pm 3.68 \\
(19.4-33.5)\end{array}$ & $\begin{array}{c}22.18 \pm 2.9 \\
(16.6-28.6)\end{array}$ & $\begin{array}{l}24.34 \pm 2.76 \\
(19.8-28.7)\end{array}$ & $\begin{array}{l}24.12 \pm 2.74 \\
(19.3-30.4)\end{array}$ & $\begin{array}{l}25.03 \pm 3.56 \\
(18.1-30.4)\end{array}$ & $\begin{array}{c}24.62 \pm 3.2 \\
(20-36.2)\end{array}$ & $\begin{array}{c}22.8 \pm 2.28 \\
(18.9-28.1)\end{array}$ & $1>7,3,8,5,6,2>94$ \\
\hline $\begin{array}{l}\text { BMI (Body Mass } \\
\text { Index) }\left[\mathrm{kg} / \mathrm{m}^{2}\right]\end{array}$ & $\begin{array}{l}24.67 \pm 2.68 \\
(20.9-30.8)\end{array}$ & $\begin{array}{l}21.06 \pm 1.83 \\
(18.5-25.2)\end{array}$ & $\begin{array}{l}23.59 \pm 4.99 \\
(17.6-36.2)\end{array}$ & $\begin{array}{c}19.38 \pm 1.99 \\
(15.1-25)\end{array}$ & $\begin{array}{l}24.74 \pm 5.05 \\
(17.8-40.2)\end{array}$ & $\begin{array}{l}21.25 \pm 2.81 \\
(16.6-29.3)\end{array}$ & $\begin{array}{c}24.09 \pm 3.95 \\
(17-33.4)\end{array}$ & $\begin{array}{c}22.5 \pm 2.62 \\
(18.1-31.3)\end{array}$ & $\begin{array}{c}22 \pm 4.33 \\
(15.3-33.4)\end{array}$ & $5,1,7,3,8,9,6,2>4$ \\
\hline $\begin{array}{l}\text { PBF (Percent Body } \\
\text { Fat) [\%] }\end{array}$ & $\begin{array}{c}30.7 \pm 6.8 \\
(9.7-41.5)\end{array}$ & $\begin{array}{l}25.55 \pm 5.06 \\
(16.2-36.1)\end{array}$ & $\begin{array}{c}29.86 \pm 8.35 \\
(18-49.9)\end{array}$ & $\begin{array}{c}22.53 \pm 6.4 \\
(13.2-36.3)\end{array}$ & $\begin{array}{c}32.19 \pm 8.4 \\
(19.8-52)\end{array}$ & $\begin{array}{c}25 \pm 7.38 \\
(9.7-40.5)\end{array}$ & $\begin{array}{l}30.48 \pm 7.52 \\
(14.2-48.1)\end{array}$ & $\begin{array}{l}28.44 \pm 5.13 \\
(19.1-38.2)\end{array}$ & $\begin{array}{l}28.96 \pm 8.46 \\
(13.5-48.1)\end{array}$ & $5,1,7,3,9,8,2,6>4$ \\
\hline $\begin{array}{l}\text { WHR (Waist-Hip } \\
\text { Ratio) }\end{array}$ & $\begin{array}{c}0.87 \pm 0.06 \\
(0.75-0.96)\end{array}$ & $\begin{array}{c}0.84 \pm 0.05 \\
(0.78-0.98)\end{array}$ & $\begin{array}{c}0.87 \pm 0.07 \\
(0.77-1.04)\end{array}$ & $\begin{array}{l}0.82 \pm 0.03 \\
(0.78-0.9)\end{array}$ & $\begin{array}{c}0.87 \pm 0.05 \\
(0.79-0.97)\end{array}$ & $\begin{array}{c}0.83 \pm 0.05 \\
(0.75-0.96)\end{array}$ & $\begin{array}{l}0.88 \pm 0.04 \\
(0.8-0.94)\end{array}$ & $\begin{array}{c}0.85 \pm 0.04 \\
(0.78-0.95)\end{array}$ & $\begin{array}{c}0.85 \pm 0.06 \\
(0.74-1.02)\end{array}$ & $7,5,3,1>9,8,2,6,4$ \\
\hline $\begin{array}{l}\text { VFL (Visceral Fat } \\
\text { Level) [kg] }\end{array}$ & $\begin{array}{l}9 \pm 3.57 \\
(2-16)\end{array}$ & $\begin{array}{c}5.73 \pm 2.15 \\
(3-13)\end{array}$ & $\begin{array}{c}8.72 \pm 5.74 \\
(3-24)\end{array}$ & $\begin{array}{c}4.64 \pm 2.1 \\
(2-10)\end{array}$ & $\begin{array}{c}9.36 \pm 5.24 \\
(3-23)\end{array}$ & $\begin{array}{c}5.96 \pm 3.27 \\
(2-16)\end{array}$ & $\begin{array}{c}8.72 \pm 4.08 \\
(2-19)\end{array}$ & $\begin{array}{c}7.2 \pm 2.92 \\
(3-17)\end{array}$ & $\begin{array}{c}7.64 \pm 4.63 \\
(2-20)\end{array}$ & $5,1,7,3,9,8>6,2>4$ \\
\hline Target Weight $[\mathrm{kg}]$ & $\begin{array}{l}62.25 \pm 5.12 \\
(53.6-70.8)\end{array}$ & $\begin{array}{c}59.57 \pm 4.4 \\
(52.3-68.9)\end{array}$ & $\begin{array}{l}60.81 \pm 6.57 \\
(50.9-78.7)\end{array}$ & $\begin{array}{l}58.65 \pm 4.12 \\
(49.7-65.1)\end{array}$ & $\begin{array}{l}59.79 \pm 4.87 \\
(52.3-66.8)\end{array}$ & $\begin{array}{l}60.58 \pm 4.12 \\
(53.6-69.8)\end{array}$ & $\begin{array}{c}60.8 \pm 5.52 \\
(52.3-70.6)\end{array}$ & $\begin{array}{l}61.45 \pm 5.86 \\
(54.3-84.2)\end{array}$ & $\begin{array}{c}59.45 \pm 2.94 \\
(54.3-66)\end{array}$ & $1,8,3,7,6,5,2,9,4$ \\
\hline Weight Control [kg] & $\begin{array}{l}-6.95 \pm 5.89 \\
(-19.5-1.7)\end{array}$ & $\begin{array}{l}1.23 \pm 5.06 \\
(-10.8-8.3)\end{array}$ & $\begin{array}{c}-5.02 \pm 11.8 \\
(-37.2-10.5)\end{array}$ & $\begin{array}{l}5.77 \pm 5.27 \\
(-8.9-16.2)\end{array}$ & $\begin{array}{c}-6.94 \pm 11 \\
(-36.4-10.1)\end{array}$ & $\begin{array}{l}1.24 \pm 7.36 \\
(-19.3-14)\end{array}$ & $\begin{array}{c}-5.47 \pm 8.64 \\
(-27.9-11.6)\end{array}$ & $\begin{array}{l}-1.81 \pm 5.83 \\
(-20.7-9.8)\end{array}$ & $\begin{array}{c}-0.72 \pm 10.58 \\
(-27.9-17)\end{array}$ & $4>6,2,9,8>3,7,5,1$ \\
\hline BFM Control [kg] & $\begin{array}{l}-7.63 \pm 5.74 \\
(-19.5-1.7)\end{array}$ & $\begin{array}{l}-1.45 \pm 3.95 \\
(-12.1-3.8)\end{array}$ & $\begin{array}{c}-6.87 \pm 10.57 \\
(-37.2-4.8)\end{array}$ & $\begin{array}{l}1.28 \pm 4.22 \\
(-8.9-7.9)\end{array}$ & $\begin{array}{l}-8.63 \pm 9.93 \\
(-36.4-3.9)\end{array}$ & $\begin{array}{l}-1.65 \pm 6.03 \\
(-19.3-7.9)\end{array}$ & $\begin{array}{l}-6.94 \pm 7.57 \\
(-27.9-6.5)\end{array}$ & $\begin{array}{c}-4.24 \pm 5 \\
(-20.7-3.4)\end{array}$ & $\begin{array}{l}-4.54 \pm 8.75 \\
(-27.9-7.9)\end{array}$ & $4>2,6,8,9>3,7,1,5$ \\
\hline FFM Control [kg] & $\begin{array}{c}0.68 \pm 1.04 \\
(0-3.7)\end{array}$ & $\begin{array}{c}2.68 \pm 2.47 \\
(0-7.3)\end{array}$ & $\begin{array}{c}1.84 \pm 2.12 \\
(0-6)\end{array}$ & $\begin{array}{c}4.49 \pm 3.23 \\
(0-10.5)\end{array}$ & $\begin{array}{c}1.7 \pm 1.76 \\
(0-6.2)\end{array}$ & $\begin{array}{c}2.88 \pm 2.58 \\
(0-8.6)\end{array}$ & $\begin{array}{c}1.47 \pm 2.35 \\
(0-7.3)\end{array}$ & $\begin{array}{c}2.42 \pm 2.48 \\
(0-7.6)\end{array}$ & $\begin{array}{c}3.82 \pm 2.85 \\
(0-9.1)\end{array}$ & $4,9>6,2,8,3,5,7>1$ \\
\hline Obesity Degree & $\begin{array}{c}114.68 \pm 12.46 \\
(97-143)\end{array}$ & $\begin{array}{c}98.08 \pm 8.38 \\
(86-117)\end{array}$ & $\begin{array}{c}110.88 \pm 22.54 \\
(82-168)\end{array}$ & $\begin{array}{c}90.04 \pm 9.26 \\
(70-116)\end{array}$ & $\begin{array}{c}115.68 \pm 23.55 \\
(83-187)\end{array}$ & $\begin{array}{c}97.69 \pm 12.47 \\
(77-136)\end{array}$ & $\begin{array}{c}114.64 \pm 17.82 \\
(79-156)\end{array}$ & $\begin{array}{c}104.68 \pm 12.18 \\
(84-146)\end{array}$ & $\begin{array}{c}102.4 \pm 20.12 \\
(71-156)\end{array}$ & $5,1,7>3,8,9,2,6>4$ \\
\hline
\end{tabular}

Table 2 - Physiological parameters in women performing various types of physical activity

\begin{tabular}{|c|c|c|c|c|c|c|c|c|c|c|}
\hline \multirow[t]{2}{*}{ Parameter } & Golf & Martial arts & Aerobic & Jogging & Swimming & General P.E. & Sauna & Cycling & $\begin{array}{l}\text { Individual } \\
\text { training }\end{array}$ & Differences \\
\hline & 1 & 2 & 3 & 4 & 5 & 6 & 7 & 8 & 9 & \\
\hline HRmin [bpm] & $\begin{array}{c}84.12 \pm 7.81 \\
(72-100)\end{array}$ & $\begin{array}{c}126.5 \pm 6.49 \\
(114-149)\end{array}$ & $\begin{array}{c}100.28 \pm 10.69 \\
(83-130)\end{array}$ & $\begin{array}{l}122.8 \pm 5.8 \\
(108-133)\end{array}$ & $\begin{array}{c}121.28 \pm 9.52 \\
(105-149)\end{array}$ & $\begin{array}{c}108.77 \pm 9.65 \\
(81-121)\end{array}$ & $\begin{array}{c}106.72 \pm 5.7 \\
(98-118)\end{array}$ & $\begin{array}{c}102.48 \pm 15.51 \\
(78-124)\end{array}$ & $\begin{array}{c}92.32 \pm 12.55 \\
(74-125)\end{array}$ & $2>4,5>6,7>8,3>9>1$ \\
\hline HRavg [bpm] & $\begin{array}{c}67.24 \pm 4.94 \\
(56-75)\end{array}$ & $\begin{array}{l}82.81 \pm 7.98 \\
(70-97)\end{array}$ & $\begin{array}{c}73.8 \pm 7.13 \\
(63-89)\end{array}$ & $\begin{array}{l}80.96 \pm 10.52 \\
(62-102)\end{array}$ & $\begin{array}{c}81.64 \pm 6.66 \\
(71-97)\end{array}$ & $\begin{array}{c}75.5 \pm 7.12 \\
(59-88)\end{array}$ & $\begin{array}{c}86.2 \pm 6.73 \\
(69-97)\end{array}$ & $\begin{array}{l}73 \pm 8.49 \\
(60-85)\end{array}$ & $\begin{array}{c}70.64 \pm 6.82 \\
(60-86)\end{array}$ & $7>2,5,4>6,3,8,9,1$ \\
\hline HRmax [bpm] & $\begin{array}{c}114.32 \pm 10.06 \\
(99-136)\end{array}$ & $\begin{array}{c}159.19 \pm 10.88 \\
(144-181)\end{array}$ & $\begin{array}{c}134.64 \pm 16.14 \\
(109-168)\end{array}$ & $\begin{array}{c}156.48 \pm 10.57 \\
(143-181)\end{array}$ & $\begin{array}{c}148.32 \pm 12.56 \\
(128-177)\end{array}$ & $\begin{array}{c}147.38 \pm 12.79 \\
(120-168)\end{array}$ & $\begin{array}{l}130.8 \pm 6.6 \\
(118-140)\end{array}$ & $\begin{array}{l}132 \pm 18.93 \\
(107-163)\end{array}$ & $\begin{array}{c}125.48 \pm 29.36 \\
(12-168)\end{array}$ & $2,4>5,6>3,8,7,9>1$ \\
\hline Recovery time [h] & $\begin{array}{c}0.48 \pm 0.77 \\
(0-3)\end{array}$ & $\begin{array}{c}12.77 \pm 3.54 \\
(6-26)\end{array}$ & $\begin{array}{c}2.8 \pm 2.87 \\
(0-13)\end{array}$ & $\begin{array}{c}10.48 \pm 2.28 \\
(5-14)\end{array}$ & $\begin{array}{c}9.44 \pm 5.27 \\
(4-26)\end{array}$ & $\begin{array}{c}5.23 \pm 2.75 \\
(1-11)\end{array}$ & $\begin{array}{c}1.16 \pm 0.47 \\
(0-2)\end{array}$ & $\begin{array}{c}4.32 \pm 3.78 \\
(0-11)\end{array}$ & $\begin{array}{c}1.88 \pm 2.39 \\
(0-10)\end{array}$ & $2>4>5>6,8,3,9,7>1$ \\
\hline $\begin{array}{l}\text { PTE-Peak Training } \\
\text { Effect }\end{array}$ & $\begin{array}{c}1.24 \pm 0.21 \\
(1-1.8)\end{array}$ & $\begin{array}{c}2.92 \pm 0.46 \\
(2.1-4.3)\end{array}$ & $\begin{array}{c}1.78 \pm 0.53 \\
(1.2-3)\end{array}$ & $\begin{array}{l}2.7 \pm 0.3 \\
(2.1-3.1)\end{array}$ & $\begin{array}{c}2.45 \pm 0.64 \\
(1.7-4.3)\end{array}$ & $\begin{array}{c}2.28 \pm 0.51 \\
(1.3-3.9)\end{array}$ & $\begin{array}{l}1.6 \pm 0.25 \\
(1.3-2.1)\end{array}$ & $\begin{array}{c}1.96 \pm 0.68 \\
(1.1-3)\end{array}$ & $\begin{array}{c}1.66 \pm 0.55 \\
(1-3.2)\end{array}$ & $2>4>5,6,8,3,9,7>1$ \\
\hline $\begin{array}{l}\text { Energy expenditure } \\
\text { [kcal] }\end{array}$ & $\begin{array}{c}240.92 \pm 43.88 \\
(171-329)\end{array}$ & $\begin{array}{c}638.38 \pm 43.91 \\
(542-709)\end{array}$ & $\begin{array}{c}350.48 \pm 68.05 \\
(237-472)\end{array}$ & $\begin{array}{c}595.88 \pm 51.62 \\
(506-688)\end{array}$ & $\begin{array}{c}553.88 \pm 53.87 \\
(447-679)\end{array}$ & $\begin{array}{c}434.04 \pm 99.91 \\
(235-580)\end{array}$ & $\begin{array}{c}121.44 \pm 12.37 \\
(96-144)\end{array}$ & $\begin{array}{c}399.92 \pm 121.78 \\
(201-585)\end{array}$ & $\begin{array}{c}297.28 \pm 85.16 \\
(186-498)\end{array}$ & $2>4>5>6,8,3>9>1>7$ \\
\hline VO2avg $[\mathrm{mL} / \mathrm{kg} / \mathrm{min}]$ & $\begin{array}{c}7.88 \pm 2.37 \\
(5-14)\end{array}$ & $\begin{array}{c}22.81 \pm 1.74 \\
(20-26)\end{array}$ & $\begin{array}{c}12.56 \pm 4.25 \\
(6-23)\end{array}$ & $\begin{array}{c}20.64 \pm 2.16 \\
\quad(17-24)\end{array}$ & $\begin{array}{c}19.8 \pm 2.72 \\
(14-25)\end{array}$ & $\begin{array}{c}16.38 \pm 5.15 \\
(6-33)\end{array}$ & $\begin{array}{c}18.2 \pm 3.24 \\
(12-24)\end{array}$ & $\begin{array}{c}14.08 \pm 4.95 \\
(7-20)\end{array}$ & $\begin{array}{c}10.44 \pm 3.95 \\
(6-21)\end{array}$ & $2>4,5,7,6>8,3>9>1$ \\
\hline $\operatorname{VO} 2 \mathrm{max}[\mathrm{mL} / \mathrm{kg} / \mathrm{min}]$ & $\begin{array}{c}18.36 \pm 3.7 \\
(13-26)\end{array}$ & $\begin{array}{c}33.31 \pm 3.97 \\
(25-45)\end{array}$ & $\begin{array}{c}26.2 \pm 5.41 \\
(17-39)\end{array}$ & $\begin{array}{c}32.56 \pm 4.82 \\
(25-48)\end{array}$ & $\begin{array}{c}28.84 \pm 4.78 \\
(21-37)\end{array}$ & $\begin{array}{c}30.88 \pm 4.55 \\
(22-41)\end{array}$ & $\begin{array}{c}26.08 \pm 2.29 \\
(22-30)\end{array}$ & $\begin{array}{c}26.08 \pm 6.24 \\
(18-37)\end{array}$ & $\begin{array}{c}24.32 \pm 7.45 \\
(11-39)\end{array}$ & $2>4,6>5,3,8,7,9>1$ \\
\hline EPOCavg $[\mathrm{mL} / \mathrm{kg}]$ & $\begin{array}{c}2.6 \pm 1.26 \\
(1-5)\end{array}$ & $\begin{array}{c}25.88 \pm 4.66 \\
(17-36)\end{array}$ & $\begin{array}{c}7.36 \pm 6.96 \\
(2-32)\end{array}$ & $\begin{array}{c}21.13 \pm 6.8 \\
(8-33)\end{array}$ & $\begin{array}{c}17.92 \pm 8.98 \\
(9-49)\end{array}$ & $\begin{array}{c}10.96 \pm 4.5 \\
(2-21)\end{array}$ & $\begin{array}{c}4.17 \pm 1.86 \\
(2-10)\end{array}$ & $\begin{array}{c}9.48 \pm 6.73 \\
(2-21)\end{array}$ & $\begin{array}{c}5.68 \pm 5.19 \\
(2-22)\end{array}$ & $2>4>5>6,8,3,9,7>1$ \\
\hline EPOCmax [mL/kg] & $\begin{array}{c}5.52 \pm 2.92 \\
(2-12)\end{array}$ & $\begin{array}{c}47.12 \pm 13.12 \\
\quad(27-74)\end{array}$ & $\begin{array}{c}15.52 \pm 15.16 \\
\quad(4-65)\end{array}$ & $\begin{array}{l}40 \pm 9.07 \\
(24-57)\end{array}$ & $\begin{array}{c}27.32 \pm 13.31 \\
(12-58)\end{array}$ & $\begin{array}{c}24.81 \pm 11.72 \\
\quad(4-52)\end{array}$ & $\begin{array}{c}9.2 \pm 3.44 \\
(5-18)\end{array}$ & $\begin{array}{c}20.84 \pm 15.67 \\
\quad(2-48)\end{array}$ & $\begin{array}{c}12.92 \pm 12.75 \\
\quad(3-61)\end{array}$ & $2>4>5,6,8,3,9,7>1$ \\
\hline $\begin{array}{l}\text { Respiratory rateavg } \\
\text { [brpm] }\end{array}$ & $\begin{array}{r}16 \pm 1.61 \\
(13-18)\end{array}$ & $\begin{array}{c}23.42 \pm 1.21 \\
(21-26)\end{array}$ & $\begin{array}{c}18.76 \pm 1.54 \\
(17-22)\end{array}$ & $\begin{array}{c}22.76 \pm 1.42 \\
(20-27)\end{array}$ & $\begin{array}{c}21.65 \pm 1.47 \\
(20-26)\end{array}$ & $\begin{array}{c}20.08 \pm 1.52 \\
(16-22)\end{array}$ & $\begin{array}{c}18.28 \pm 0.98 \\
(17-21)\end{array}$ & $\begin{array}{c}19.68 \pm 2.38 \\
(15-24)\end{array}$ & $\begin{array}{c}17.64 \pm 2.38 \\
(13-24)\end{array}$ & $2,4,5>6,8,3,7,9>1$ \\
\hline $\begin{array}{l}\text { Respiratory ratemax } \\
\text { [brpm] }\end{array}$ & $\begin{array}{c}23.6 \pm 2.18 \\
(18-27)\end{array}$ & $\begin{array}{c}35.23 \pm 4.16 \\
(27-41)\end{array}$ & $\begin{array}{c}28.32 \pm 6.7 \\
(23-54)\end{array}$ & $\begin{array}{l}35.12 \pm 4 \\
(28-41)\end{array}$ & $\begin{array}{c}29.92 \pm 4.76 \\
(25-39)\end{array}$ & $\begin{array}{c}31.73 \pm 6.69 \\
(23-54)\end{array}$ & $\begin{array}{c}25.08 \pm 1.73 \\
(23-29)\end{array}$ & $\begin{array}{l}30.12 \pm 5.71 \\
(22-44)\end{array}$ & $\begin{array}{c}26.72 \pm 5.05 \\
(19-42)\end{array}$ & $2,4>6,8,5,3>9,7>1$ \\
\hline
\end{tabular}




\begin{tabular}{|c|c|c|c|c|c|c|c|c|c|c|}
\hline \multicolumn{11}{|l|}{ Exercise intensity [min] } \\
\hline Easy < 107 [bpm] & $\begin{array}{c}54: 18 \pm 6: 27 \\
(39: 34-60: 00)\end{array}$ & $\begin{array}{c}7: 31 \pm 5: 32 \\
(0: 25-21: 03)\end{array}$ & $\begin{array}{l}43: 21 \pm 13: 41 \\
(10: 03-60: 00)\end{array}$ & $\begin{array}{c}9: 46 \pm 7: 15 \\
(0: 45-28: 05)\end{array}$ & $\begin{array}{c}8: 53 \pm 8: 24 \\
(0: 44-30: 55)\end{array}$ & $\begin{array}{l}28: 49 \pm 14: 06 \\
(8: 25-59: 43)\end{array}$ & $\begin{array}{c}6: 52 \pm 2: 39 \\
(3: 06-12: 56)\end{array}$ & $\begin{array}{l}35: 43 \pm 19: 58 \\
(6: 32-60: 00)\end{array}$ & $\begin{array}{l}48: 19 \pm 12: 11 \\
(23: 22-60: 00)\end{array}$ & $1>9,3>8,6>4,5,2,7$ \\
\hline $\begin{array}{l}\text { Moderate 107-124 } \\
\text { [bpm] }\end{array}$ & $\begin{array}{c}5: 09 \pm 6: 00 \\
(0: 00-20: 06)\end{array}$ & $\begin{array}{l}15: 50 \pm 7: 03 \\
(4: 11-37: 18)\end{array}$ & $\begin{array}{c}11: 01 \pm 8: 34 \\
(0: 00-28: 06)\end{array}$ & $\begin{array}{c}18: 12 \pm 7: 02 \\
(4: 43-37: 18)\end{array}$ & $\begin{array}{c}26: 54 \pm 9: 55 \\
(10: 58-43: 25)\end{array}$ & $\begin{array}{c}18: 43 \pm 8: 45 \\
(0: 17-33: 21)\end{array}$ & $\begin{array}{c}5: 53 \pm 2: 13 \\
(1: 02-10: 08)\end{array}$ & $\begin{array}{l}12: 53 \pm 10: 22 \\
(0: 00-36: 31)\end{array}$ & $\begin{array}{c}7: 33 \pm 7: 46 \\
(0: 00-31: 09)\end{array}$ & $5>6,4,2,8,3>9,7,1$ \\
\hline Difficult 125-141 [bpm] & $\begin{array}{l}0: 33 \pm 0: 57 \\
(0: 00-3: 01)\end{array}$ & $\begin{array}{c}27: 20 \pm 8: 39 \\
(10: 29-42: 17)\end{array}$ & $\begin{array}{c}4: 53 \pm 7: 08 \\
(0: 00-20: 42)\end{array}$ & $\begin{array}{c}25: 53 \pm 9: 12 \\
(10: 29-41: 34)\end{array}$ & $\begin{array}{l}21: 43 \pm 8: 51 \\
(8: 58-43: 42)\end{array}$ & $\begin{array}{c}10: 55 \pm 7: 26 \\
(0: 00-24: 31)\end{array}$ & $\begin{array}{c}1: 15 \pm 1: 47 \\
(0: 00-5: 56)\end{array}$ & $\begin{array}{c}9: 12 \pm 9: 42 \\
(0: 00-28: 45)\end{array}$ & $\begin{array}{c}3: 10 \pm 4: 29 \\
(0: 00-13: 23)\end{array}$ & $2,4>5>6,8>3,9,7,1$ \\
\hline $\begin{array}{l}\text { Very Difficult 142-159 } \\
\text { [bpm] }\end{array}$ & $\begin{array}{l}0: 00 \pm 0: 00 \\
(0: 00-0: 00)\end{array}$ & $\begin{array}{c}8: 55 \pm 6: 27 \\
(0: 03-22: 52)\end{array}$ & $\begin{array}{c}0: 45 \pm 2: 08 \\
(0: 00-10: 17)\end{array}$ & $\begin{array}{c}5: 42 \pm 3: 56 \\
(0: 00-11: 09)\end{array}$ & $\begin{array}{c}2: 26 \pm 4: 52 \\
(0: 00-22: 52)\end{array}$ & $\begin{array}{c}1: 33 \pm 2: 14 \\
(0: 00-7: 25)\end{array}$ & $\begin{array}{l}0: 00 \pm 0: 00 \\
(0: 00-0: 00)\end{array}$ & $\begin{array}{c}2: 12 \pm 3: 26 \\
(0: 00-14: 05)\end{array}$ & $\begin{array}{c}0: 57 \pm 3: 17 \\
(0: 00-16: 19)\end{array}$ & $2>4>5,8,6,9,3,7,1$ \\
\hline Maximal $\geq 160[\mathrm{bpm}]$ & $\begin{array}{l}0: 00 \pm 0: 00 \\
(0: 00-0: 00)\end{array}$ & $\begin{array}{l}0: 24 \pm 0: 58 \\
(0: 00-4: 12)\end{array}$ & $\begin{array}{l}0: 00 \pm 0: 00 \\
(0: 00-0: 00)\end{array}$ & $\begin{array}{l}0: 28 \pm 1: 11 \\
(0: 00-4: 12)\end{array}$ & $\begin{array}{l}0: 03 \pm 0: 15 \\
(0: 00-1: 12)\end{array}$ & $\begin{array}{c}0: 00 \pm 0: 00 \\
(0: 00-0: 00)\end{array}$ & $\begin{array}{l}0: 00 \pm 0: 00 \\
(0: 00-0: 00)\end{array}$ & $\begin{array}{l}0: 01 \pm 0: 03 \\
(0: 00-0: 14)\end{array}$ & $\begin{array}{c}0: 00 \pm 0: 00 \\
(0: 00-0: 00)\end{array}$ & $4,2>5,8,9,7,6,3,1$ \\
\hline
\end{tabular}

\section{Discussion}

This study sought to evaluate the effectiveness of various forms of PA in physical education programs offered by universities in Poland, Hungary, and the United Kingdom.

The results indicate that martial arts, JFBS, and swimming were the most physically intensive types of PA among female university students, whereas playing golf involved the lowest levels of physical exertion. The research studies investigating the relationships between PA and performance suggest that higher levels of PA have a positive impact on health-related physical fitness of individual ${ }^{29}$ including children and adolescents $s^{30,31}$. The current study results indicated that the average values of body mass, BMI, BFM, PBF, WHR, VFL, and obesity degree were significantly $(p<0.05)$ lower among the participants who jogged, performed martial arts, and attended PE classes. In contrast, the average body mass of students who played golf was significantly higher than in other PA groups. Furthermore, the data show that physiological parameters were the highest in the martial arts group, followed by JFBS, whereas participants who played golf and trained individually observed the lowest values of physiological indicators. This might be because the slimmer participants tended to select more vigorous forms of exercise, whereas heavier students were more likely to choose the moderate forms of PA. This finding is consistent with the results from the previous study by Podstawski et al. ${ }^{32}$ suggesting that choice of PA could be influenced by height and body mass. In the article, Podstawski et al. ${ }^{32}$ reported that taller and heavier participants preferred moderate PA, such as golf, aerobics, or general PA, whereas intense forms of PA like martial arts and JFBS were favoured by slimmer students. Another explanation could be that exercising under the supervision of qualified and experienced instructors is more effective and provides greater health and fitness benefits than individual training. Although King ${ }^{33}$ suggests that individual voluntary training can effectively improve fitness levels and decrease body mass, he also notices that those who drop out after the first two or three months are more likely to return to their baseline weight or even gain weight (yo-yo effect).

The results of this study support the notion of the effectiveness of the organised training sessions. Since not all students can effectively plan and implement their training routines, they require coaches, instructors, or PE teachers who encourage them to improve their PA levels, motor fitness, and overall health ${ }^{34-36}$. Evidence shows that organised training sessions and PE classes are fun and effective in forming health and fitness habits in students ${ }^{37}$. In contrast, ineffective use of workout time might decrease students' motivation to participate in sports and $\mathrm{PA}^{38}$. There are many socio-demographic, psychological, and environmental factors, such as gender, health, habits, lifestyle, motivation, willpower that influence decision to participate in $\mathrm{PA}^{39}$. Neupert et al. ${ }^{40}$ propose that $30-50 \%$ of individuals who enroll in a training program quit after two or three months. Dropout rates tend to be higher among people with a sedentary lifestyle, and university students who generally fall into this category $^{39}$. However, those who continue training after the first six months are more likely to form a fitness habit and an active lifestyle ${ }^{41}$. With regards to somatic traits and body composition parameters, the analyses of WHR values revealed a higher prevalence of android obesity among students who participated in aerobics, golfing, and swimming classes ( 0.87 in each group). It is worth noticing that even though swimming is regarded as a cyclic and energy-intensive sports discipline $(553.9 \mathrm{kcal} / \mathrm{h})$, the female participants who practiced swimming recorded the highest values of BMI $\left(24.7 \mathrm{~kg} / \mathrm{m}^{2}\right)$, BFM $(22.4 \mathrm{~kg})$, and PBF $(32.2 \%)$ in the research study sample. Much research has focused on exploring the impact of swimming on the human body. A previous study by Podstawski et al., $(2015)^{32}$ demonstrated that swimming tended to be selected as a form of PA by overweight women. Gwinup ${ }^{42}$ and Jang et al. ${ }^{43}$ studies revealed that despite the similar daily energy expenditure (male swimmers - 3380 $\mathrm{kcal}$, male runners $-3460 \mathrm{kcal}$, female swimmers $-2490 \mathrm{kcal}$, female runners $-2040 \mathrm{kcal}$ ), swimmers had higher body fat levels ( $12 \%$ in men, $20 \%$ in women) than runners ( $7 \%$ in men, $15 \%$ in women). Correspondingly, Flynn et al..$^{44}$ reported similarities in the energy expenditure during 45 minutes of swimming and running at $75-80 \% \mathrm{VO}_{\text {max }}$ intensity. These results together with the findings from the current study might suggest that swimming is not as efficient in reducing body mass. Jang et al ${ }^{43}$ proposed that this could be attributed to the fact that swimmers experience more intense feelings of hunger than runners after training. Swimmers also tend to be less physically active than runners between training sessions. Temperature is another factor that influences the metabolic rate. A study conducted by White et al. ${ }^{45}$ reported that energy expenditure was $44 \%$ higher among participants who trained for 45 minutes in cold water $\left(20^{\circ} \mathrm{C}\right)$ 
than those who swam in temperature-neutral water $\left(30^{\circ} \mathrm{C}\right)$.

The study also shows that the average values of proteins, minerals, BFM, FFM, and SMM were significantly higher among participants who played golf, whereas joggers and martial arts students observed the lowest obesity levels, BFM, FFM, SMM, BMI, PBF, and VFL. Moreover, female students who enrolled in martial arts, JFBS, and PE classes recorded lower body fat levels compared to those participating in golfing, cycling, individual training, and aerobics.

This study has some limitations. First, the cross-sectional design impairs cause-effect inferences. Second, the use of Suunto Ambit 3 Peak Sapphire heart rate monitors allowed measuring only estimated values of the physiological parameters of the participants. However, it should be noted this type of device is widely used to analyze physical exertion levels in both performance and health training. Furthermore, other types of measuring equipment could not be used as effectively in this study, since it was conducted on a large and homogenous sample.

Despite these issues, a considerable strength of this investigation is the novelty of the research topic. It is one of the few studies that evaluate the effectiveness of different forms of PA among university students. Furthermore, to date, no study has examined the effectiveness of various forms of PA among female students in PE programs in higher education. The results of this investigation have practical implications for PE practitioners at all levels of education, from primary school to university. We recommend that the practitioners should focus on developing the physical fitness of students by increasing the intensity of physical exercise and levels of physical activity.

\section{Conclusions}

The results of this study corroborate the conclusions from the first stage of our research project evaluating the correlations between somatic parameters and different types of PA, as well as the relationship between specific forms of PA and the motor abilities of university students. Firstly, the current study proposes that martial arts, JFBS, and swimming were the most physically intensive types of PA among female university students, whereas playing golf required the lowest levels of physical exertion. Secondly, the results suggest that female students enrolled in martial arts, JFBS, and general PE classes were characterized by the lowest body fat levels, whereas women participating in swimming training had the highest body fat levels. Lastly, the study indicates that golf and individual training without formal supervision were less intensive and thus less effective forms of PA among the participants. Therefore, the research recommends that organized PA sessions supervised by qualified instructors are more effective than individual training.

\section{References}

1. Kremer MM, Reichert FF, Hallal PC. Intensity and duration of physical efforts in physical education classes. Rev Saúde Públ. 2012; 46(2): 320-326.

2. Garber CE, Blissmer B, Deschenes MR, Franklin BA, Lamonte MJ, Lee IM, et al. American College of Sports Medicine position stand. Quantity and quality of exercise for developing and maintaining cardiorespiratory, musculoskeletal, and neuromotor fitness in apparently healthy adults: guidance for prescribing exercise. Med Sci Sports Exerc. 2011; 43(7): 1334-1359

3. Klika B, Jordan C. High-intensity circuit training using body weight: Maximum results with minimal investment. ACSM's Health Fitness J. 2013; 17(3): 8-13

4. Thompson WR. Worldwide survey of fitness trends for 2018 . ACSM's Health Fitness J. 2017; 21(6): 10-19

5. Podstawski R, Sławek M. The Influence of Political Transformation in Poland on the Functioning of the Department of Physical Education and Sport at The University of Warmia \& Mazury in Olsztyn During The Academic Years of 1998/1999 and 2010/2011. In: Sokołowska B. Public Health in the Aspect of Modern Civilization. Biała Podlaska: PSW JPII Press. 2012: 266-278.

6. Trost SG, van der Mars H. Why we should not cut P.E.? Educ Leadership. 2010; 67(4): 60-65.

7. Gordon-Larsen P, Adair LS, Nelson MC, Popkin BM. Five-year obesity incidence in the transition period between adolescence and adulthood: the National longitudinal study of Adolescent Health. Am J Clin Nutr. 2004; 80: 569-575.

8. Kann L, Warren CW, Harris WA, Collins JL, Williams BI, Ross JG, et al. Youth risk behavior surveillance- the United States, 1995. J Sch Health. 1996; 66(10): 365-377.

9. Douglas KA, Collins JL, Warren C, Kann L, Gold R, Clayton S, et al. Results from the 1995 national college health risk behavior survey. J Am Coll Health. 1997; 46(2): 55-67.

10. Bray SR, Born HA. Transition to University and vigorous physical activity: implications for health and physiological well-being. J Am Coll Health. 2004; 52: 181-188.

11. Serlachius A, Hamer M, Wardle J. Stress, and weight change in university students in the United Kingdom. Physiol Behav. 2007; 92: 548-553.

12. Mokdad AH, Serdula MK, Dietz WH, Bowman BA, Marks JS, Koplan JP. The spread of the obesity epidemic in the United States, 1991-1998. JAMA. 1999; 282: 1519-1522.

13. Haase A, Steptoe A, Sallis JF, Wardle J. Leisure-time physical activity in university students from 23 countries: associations with health beliefs, risk awareness, and national economic development. Prev Med. 2004; 39(1): 182-190.

14. Vella-Zarb RA, Elgar FJ. The 'freshmen 5': a meta-analysis of overweight gain in the freshman year of college. J Am Coll Health. 2009; 58: 161-166.

15. Gropper SS, Gaines A, Saunders D, Clary K, Connell LJ, Simmons $\mathrm{K}$, et al. Summer doesn't reverse freshmen weight gain. FASEBJ. 2009; 23: 735.2

16. Lloyd-Richardson EE, Baily S, Fava JL, Wing R. the Tobacco Etiology Research Network (TERN). A prospective study of weight gain during the college freshman and sophomore years. Prev Med. 2009; 48: 256-261.

17. Butler SM, Black DR, Blue CL, Gretebeck RJ. Change in diet, physical activity, and body weight in female college freshmen. Am J Health Behav. 2004; 28: 24-32.

18. Hajhosseini L, Holmes T, Mohamadi P, Goudarzi V, McProud L, Hollenbeck CB. Changes in body weight, body composition, and resting metabolic rate (RMR) in first-year university students freshmen students. J Am Coll Nutr. 2006; 25: 123-127. 
19. Murphy E, Schwarzkopf R. Effects of standard set and circuit weight training on excess post-exercise oxygen consumption. J Strength Cond Res. 1992; 6(2): 66Y124.

20. Scott CB, Leighton BH, Ahearn KJ, McManus JJ. Aerobic, anaerobic, and excess postexercise oxygen consumption energy expenditure of muscular endurance and strength: 1-set of bench press to muscular fatigue. J Strength Cond Res. 2011; 25(4): 903 Y8.

21. Podstawski R, Honkanen A, Choszcz D, Boraczyński M. Maximizing university students' motor fitness by implementing a physical education program incorporating martial arts - implication study. JCSMA. 2013; 4(2): 197-205.

22. Hardman K. Physical education in schools: a global perspective. Kinesiology. 2008; 40(1), 5-28.

23. Gibson AL, Holmes JC, Desautels RL, Edmonds LB, Nuudi L. The ability of new octapolar bioimpedance spectroscopy analyzers to predict 4-component-model percentage body fat in Hispanic, black, and white adults. Am J Clin Nutr. 2008; 87(2): 332-338.

24. Podstawski R, Borysławski K, Clark CCT, Choszcz D, Finn KJ, Gronek P. Correlations between repeated use of a dry sauna for 4 x 10 minutes, physiological parameters, anthropometric features, and body composition in young sedentary and overweight men: health implications. BioMed Res Int. 2019: Article ID 7535140, 13 pages, https://doi.org/10.1155/2019/7535140

25. Podstawski R, Żurek P, Clark CCT, Laukkanen JA, Markowski P, Gronek P. A multi-factorial assessment of the 3-Minute Burpee Test. JPES, 2019; 19(2): 1083-1091.

26. Bouillod A, Cassirame J, Bousson JM, Sagawa Y Jr, Tordi N. Accuracy of the Suunto system for heart rate variability analysis during a tilt-test. Rev Bras Cineantropom Desempenho Hum. 2015; 17(4): 409-417.

27. Sandercock GR, Brodie DA. The use of heart rate variability measures to assess autonomic control during exercise. Scand J Med Sci Sports. 2006; 16(5): 302-13.

28. Valdez P, Ramírez C, García A, Talamantes J, Cortez J. Circadian and homeostatic variation in sustained attention. Chronobiol Int. 2007; 27(2): 393-416.

29. Aires L, Andersen LB, Mendonça D, Martins C, Silva G, Mota J. A 3-year longitudinal analysis of changes in fitness, physical activity, fatness, and screen time. Acta Paediatr. 2010; 99(1): 140-144

30. Martínez-Vizcaíno V, Sánchez-López M. Relationship between physical ac-tivity and physical fitness in children and adolescents. Rev Esp Cardiol. 2008; 61(2) 108-111.

31. Malina RM, Bouchard C, Bar-Or O. Growth, maturation, and physical activity. Human Kinetics Publishers: Champaign, IL. 2004

32. Podstawski R, Markowski P, Choszcz D, Klimczak J. Anthropometric indicators and motor abilities of university students performing various types of physical activities (martial arts, volleyball, bodybuilding/fitness, jogging followed by a sauna, golf, general PE classes). In: Kalina RM. Proceedings of the 1st world congress on health and martial arts in an interdisciplinary approach. Archives of Budo: Warsaw. 2015: 139-148.

33. King AC. Interventions to promote physical activity by older adults. J Gerontol: Series A. Biological Sciences and Medical Sciences. 2001; 56(2): 36-46.

34. Ball DL, Thames MH, Phelps G. Concept knowledge for teaching: what makes it special? J Teach Educ. 2008; 59(5): 389-407
35. Dodds P, Placek J, Doolittle S, Pinkham K, Ratlifle T, Portman P. Teacher/coach recruits: Background profiles, occupational decision factors, and comparisons with recruits into other physical education occupations. J Teach Phys Edu. 1992; 11: 161-176.

36. Rink JE. Effective instruction in physical education. In Silverman SJ, Ennis CD. Student learning in physical education: Applying research to enhance instruction. Human Kinetics, Champaign, Illinois. 1996: 171-198.

37. Pagnano K, Langley DJ. Teacher Perspectives on the Role of Exercise as a Management Tool in Physical Education. J Teach Phys Edu. 2001; 21: 57-74.

38. Silverman S, Scrabis KA. A review of research on instructional theory in physical education 2002-2003. J Teach Phys Educ. 2004; 41: 4-12.

39. Podstawski R, Choszcz D, Polakowska P. Effectiveness of the "Slimming Down Olsztyn Residents" health program - motivation to participate and withdraw. Baltic J Health Phys Activ. 2017; 9(2): 50-63.

40. Neupert SD, Lachman ME, Whitbourne SB. Exercise, self-efficacy, and control beliefs predict exercise behaviour after an exercise intervention for older adults. J Aging Phys Activ. 2009; 17(1): 1-16.

41. Dishman RK, Salis JF. Determinants and interventions for physical activity and exercise. In: Bouchard C, Shepard RJ, Stephens T. Physical activity, fitness, and health Human Kinetics, Champaign, IL. 1994: 214-238).

42. Gwinup G. Weight loss without dietary restriction: Efficacy of different forms of aerobic exercise. Am J Sports Med. 1987; 15(3): 275-279.

43. Jang KT, Flynn MG, Costill DL, Kirwan JP, Houmard JA, Mitchell $\mathrm{JB}$, et al. Energy balance in competitive swimmers and runners. J Swim Res. 1987; 3: 19-23.

44. Flynn ML, Costill DL, Kirwan JP, Mitchell JB, Houmard JA, Fink WJ, et al. Fat storage in athletes: metabolic and hormonal responses to swimming and running. Int J Sports Med. 1990;11: 433-440.

45. White LJ. Dressendorfer R., Holland E, McCoy SC, Ferguson MA. Increased caloric intake soon after in cold water. Int J Sport Nutr Exerc Metab. 2005; 15: 34-40.

\section{Corresponding author}

Robert Podstawski

University of Warmia and Mazury in Olsztyn, Faculty of Geoengineering, Department of Tourism, Recreation and Ecology, M. Oczapowskiego 5; 10-719 Olsztyn, Poland.

Email: podstawskirobert@gmail.com

Manuscript received on May 13, 2020

Manuscript accepted on October 26, 2020

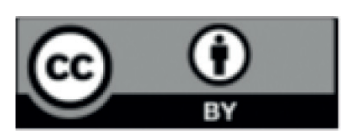

Motriz. The Journal of Physical Education. UNESP. Rio Claro, SP, Brazil - eISSN: 1980-6574 - under a license Creative Commons - Version 4.0 